\begin{tabular}{ll} 
Journal of Environmental \\
Analysis and Progress \\
ISSN: $2525-815 \mathrm{X}$ & Journal homepage: $\underline{\text { www.jeap.ufrpe.br/ }}$ \\
\hline
\end{tabular}

\title{
Efeitos da fertilização sobre interações tritróficas entre planta, afídeo e formigas invasoras: um teste experimental
}

\section{Effects of fertilization on tritrophic interactions among invasive plant, aphid and ants: an experimental test}

\author{
Renata Alexandre Bianchi ${ }^{\mathrm{a}}$, Henrique Venâncio ${ }^{\mathrm{b}}$, Guilherme Magalhães Viana ${ }^{\mathrm{a}}$, José Afonso da Silva \\ Galhardo $^{\mathrm{a}}$, Jean Carlos Santos ${ }^{\mathrm{c}}$ \\ ${ }^{a}$ Universidade Federal de Uberlândia-UFU, Instituto de Biologia, Av. Pará, n. 1720, Uberlândia, Minas Gerais, Brasil. \\ CEP: 38405-320. E-mail: renatalebianchi@gmail.com, vianagm93@gmail.com, afonso_dasilva19@ hotmail.com. \\ ${ }^{\text {b }}$ Universidade de São Paulo-USP, Faculdade de Filosofia Ciências e Letras de Ribeirão Preto, Programa de Pós- \\ Graduação em Entomologia, Av. Bandeirantes, n. 3900, Ribeirão Preto, São Paulo, Brasil. CEP: 14040-901. E-mail: \\ henrivens@gmail.com. \\ ${ }^{c}$ Universidade Federal de Sergipe-UFSE, Departamento de Ecologia, Av. Marechal Rondon, s/n, Bairro Jardim Rosa \\ Elze, São Cristóvão, Sergipe. CEP: 49100-000. E-mail: jcsantosbio@ gmail.com.
}

\begin{abstract}
ART I CLE IN F O
Recebido 08 Jul 2019

Aceito 29 Jul 2019

Publicado 31 Jul 2019

A B S T R A C T

Fertilization can trigger a positive effect on weeds, reflecting, through a cascade effect, in a populational increase of pests like aphids and mutualistic ants. In this study, it was evaluated how soil fertilization influences on the interaction among Tithonia diversifolia (Asteraceae), Aphis gossypii (Aphididae) and the ants Pheidole megacephala and Solenopsis invicta (Formicidae). Through a greenhouse experiment, 30 seedlings that had aphids and ants were separated into two groups: one nutritionally enriched with synthetic fertilizer, and another without fertilization. Foliar chlorophyll and the number of aphids and ants were sampled before and after the experiment. The results demonstrated an increase of more than $50 \%$ in foliar chlorophyll and number of aphids and ants at the beginning and end of the study. However, only chlorophyll and number of ants were higher on fertilized seedlings compared to unfertilized ones. As conclusion, our study evidences that fertilization produces a positive bottom-up effect on tritrophic interaction among invasive plants, aphids and ants.
\end{abstract}

Keywords: Bottom-up effect, pests, weed, nutritional enrichment.

\section{R E S U M O}

A fertilização pode desencadear um efeito positivo em plantas daninhas, refletindo, através de um efeito cascata, no aumento populacional de pragas como afídeos e formigas mutualistas. Neste estudo foi avaliado como a fertilização do solo influencia na interação entre as invasoras Tithonia diversifolia (Asteraceae), Aphis gossypii (Aphididae) e das formigas Pheidole megacephala e Solenopsis invicta (Formicidae). Através de um experimento, 30 plântulas que apresentavam afídeos e formigas foram separadas em dois grupos: um enriquecido nutricionalmente com fertilizante sintético, e outro sem fertilização. A clorofila foliar, o número de afídeos e de formigas foram coletados no início e término do experimento. Os resultados mostraram um aumento de mais de $50 \%$ na clorofila foliar, no número de afídeos e de formigas do início ao final do estudo no grupo fertilizado. No entanto, somente a clorofila e o número de formigas foram maiores nas plântulas fertilizadas em relação às sem fertilização. Como conclusão, nosso estudo demonstra que a fertilização causa um efeito bottom-up positivo na interação tritróficas entre plantas, afídeos e formigas invasoras.

Palavras-Chave: Efeito bottom-up, pragas, plantas daninhas, enriquecimento nutricional. 


\section{Introdução}

Atividades antrópicas são as principais causas da introdução e expansão de espécies invasoras no mundo (Stanley et al., 2013). Em ambientes agrícolas, os fertilizantes são frequentemente utilizados para impulsionar $o$ desempenho de plantas comerciais através do enriquecimento de nitrogênio $(\mathrm{N})$ do solo. Porém, a fertilização também pode beneficiar indiretamente populações de fitófagos que atacam plantas comerciais e não comerciais que foram fertilizadas (Butler, Garratt \& Leather, 2012). Pragas generalistas, como alguns afídeos, comumente interagem com outros grupos, incluindo plantas daninhas e formigas invasoras em agrossistemas. Esta interação tritrófica pode dificultar no controle desse grupo.

Plantas daninhas são capazes de absorver e alocar rapidamente macronutrientes para crescimento vegetativo (Gioria \& Osborne, 2014). Sob fertilização, algumas espécies de plantas invasoras aumentam a concentração de $\mathrm{N}$ e sofrem modificações físicas (por exemplo, diminuição da dureza foliar) (Kołodziejek, 2019; Tulloss \& Cadenasso, 2016) que favorecem o ataque de afídeos (Yao, 2014). Além do aumento da preferência, plantas ricas nutricionalmente desencadeiam um efeito bottom-up positivo no mutualismo entre afídeos e formigas ao aumentar a qualidade e/ou quantidade de honeydew, um exsudado liberado pelos afídeos e que é consumido pelas formigas (Morales \& Beal, 2006). Como resultado, há um aumento na densidade de formigas que ordenham estes afídeos com honeydew rico em carboidratos; enquanto os afídeos são favorecidos, por exemplo, com um aumento na taxa reprodutiva e proteção contra inimigos (Kaplan \& Eubanks, 2002; Yao, 2014). No entanto, alguns estudos mostraram que o enriquecimento nutricional do solo pode ser prejudicial para invasoras (Singh \& Sood, 2017), ou até mesmo o crescimento de um grupo pode não desencadear um efeito bottom-up positivo no outro (Zhou et al., 2012).

Devido às lacunas do efeito da fertilização sob interações entre invasoras, foi realizado um experimento em casa de vegetação para verificar como o enriquecimento do solo influencia na qualidade nutricional do girassol-mexicano [Tithonia diversifolia (Hemsl.) A. Gray], no ataque do afídeo-do-algodão [Aphis gossypii Glover, 1877 (Hemiptera: Aphididae)] e na preferência das formigas Pheidole megacephala Fabricius, 1793 e Solenopsis invicta Buren, 1972 (Formicidae: Myrmicinae). Nesta interação facultativa, todos os níveis causam prejuízos em agro-ecossistemas; e estudos relataram que plantas hospedeiras com alto valor nutricional aumentam o desempenho do afídeo-do-algodão (Rostami et al., 2012), refletindo em maior e mais frequente ordenha dessas formigas em outras espécies (Zhou et al., 2015). Assim, é esperado que a fertilização de $T$. diversifolia induza um efeito cascata nos demais níveis tróficos, onde plantas fertilizadas apresentarão maior concentração de $\mathrm{N}$ [avaliada indiretamente pela clorofila foliar, que é correlacionada ao N (Netto et al., 2005)], no número de afídeos e de formigas em relação às plantas não fertilizadas.

\section{Material e Métodos}

Este estudo foi realizado em março de 2017 em uma casa de vegetação ( 5 x 10 metros) da Universidade Federal de Uberlândia, Uberlândia, Minas Gerais ( $\left.18^{\circ} 53^{\prime} 10^{\prime \prime} \mathrm{S}-48^{\circ} 15^{\prime} 36^{\prime \prime} \mathrm{O}\right)$. A temperatura do local foi de $\cong 28^{\circ} \mathrm{C}$, com quatro irrigações diárias automáticas com duração de três minutos cada. Este protocolo de irrigação foi mantido tanto durante a germinação das sementes de $T$. diversifolia quanto ao longo experimento.

Um total de 30 sementes de $T$. diversifolia foram cultivadas e colocadas para germinarem em potes de polietileno $(12,7 \mathrm{~cm} \times 7 \mathrm{~cm} \times 7,5 \mathrm{~cm} ; 500$ $\mathrm{mL}$ ) com substrato orgânico Bioflora ${ }^{\circledR}$ dentro da casa de vegetação. Sessenta dias após a germinação, todas as 30 plântulas de T. diversifolia foram colonizadas espontaneamente por $A$. gossypii e pelas formigas $P$. megacephala e $S$. invicta de populações próximas a estufa. Nesse momento, estas plântulas foram mantidas nos mesmos recipientes e substratos utilizados durante a germinação, e o experimento foi iniciado. $\mathrm{O}$ experimento foi composto por dois tratamentos: um controle ( $\mathrm{n}=15$ plântulas), em que o solo não foi enriquecido nutricionalmente, e um fertilizado ( $\mathrm{n}=15$ plântulas), que recebeu a adição de fertilizante no solo. A fertilização ocorreu pela adição de $6 \mathrm{~g}$ de cápsulas do fertilizante Osmocote ${ }^{\circledR}$ (15N: 9P: 12K) no substrato de cada plântula. Essas plântulas foram dispostas de forma alternada em uma bancada durante todo o experimento, que durou 12 dias.

O número de A. gossypii e de formigas, assim como a clorofila, foram analisadas em três folhas apicais das plântulas, no primeiro e décimo segundo dia do experimento (i.e., antes e depois da fertilização). Como as folhas do girassol-mexicano senescem aproximadamente um mês após a expansão (Venâncio, Silva \& Santos, 2016), os trios de folhas analisadas ao término do experimento foram diferentes das folhas da primeira semana. Para facilitar o entendimento, chamaremos as três folhas da primeira e última semana de ambos os grupos, respectivamente, de 
"folhas da primeira semana" e "folhas da última semana".

Os espécimes de insetos das folhas na primeira semana foram quantificados diretamente nas plantas, para preservar a comunidade de afídeos e formigas instaladas até o final do estudo. Para identificação inicial das formigas, dez indivíduos de cada espécie foram coletados com pincéis de todas as plantas. Ao final do experimento, realizamos a contagem dos insetos nas folhas pós fertilização. Para isso, inserimos essas folhas individualmente em placas de Petri, as levamos para laboratório e conservamos os espécimes em álcool $70 \%$ para serem contabilizados e identificados. A identificação dos insetos foi realizada através de um estereomicroscópio $\quad\left(\mathrm{OPTON}^{\circledR}, \quad\right.$ TIM-2B). A identificação das espécies de formigas foi realizada através da chave de Baccaro et al. (2008).

Nós consideramos a clorofila das folhas da primeira e última semana como indicativo da qualidade nutricional do girassol-mexicano. A clorofila das plântulas foi coletada nas folhas da primeira e da última semana em que os insetos foram coletados.. A clorofila foi mensurada na região central de cada folha através de uma leitura do clorofilômetro SPAD 502 Plus (Konica Minolta $^{\circledR}$, Japão). Todas as medidas foram realizadas após a contabilização dos insetos; e os valores de clorofila por plântula ao início e término do estudo está representado, respectivamente, pela média aritmética das folhas da primeira e última semana.

Para testar a influência do enriquecimento nutricional na clorofila foliar, no número de formigas e de afídeos em $T$. diversifolia, antes e depois da fertilização, foi utilizada ANOVA de medidas repetidas, e testes a posteriori (Tukey HSD). O número de afídeos e de formigas foram transformados em logaritmo $\left(\log _{10}\right)$. A significância estatística foi considerada a partir de valores menores que 0,05 .

\section{Resultados}

Somente Aphis gossypii, Pheidole megacephala e Solenopsis invicta foram encontradas durante a execução do experimento. As espécies estiveram presentes nas plântulas controle e fertilizadas durante a primeira e última semana do estudo.

A clorofila foliar de $T$. diversifolia, o número de $A$. gossypii e de formigas foram semelhantes para as folhas das plântulas analisadas na primeira semana ( $p>0,4$ para todas as variáveis;
Figura 1). Na última semana, as plântulas fertilizadas apresentaram maior conteúdo de clorofila e número de formigas comparado às plântulas controle (Tabela 1 e Figura 1). O número de A. gossypii foi em média $20 \%$ maior nas plantas fertilizadas, porém não foi suficiente para diferir com o grupo controle (Figura 1b). Temporalmente, tanto o número de formigas quanto de afídeos não diferiram estatisticamente da primeira à terceira semana de estudo (Tabela 1). A fertilização quase dobrou os valores de todas as variáveis analisadas neste estudo da primeira à terceira semana (Tukey: $\mathrm{p}<0,003$; Figura 1). Além disso, o número de afídeos e de formigas das plântulas sem fertilização diminuiu em mais de $20 \%$ entre as duas semanas (Figura 1bc).

a)

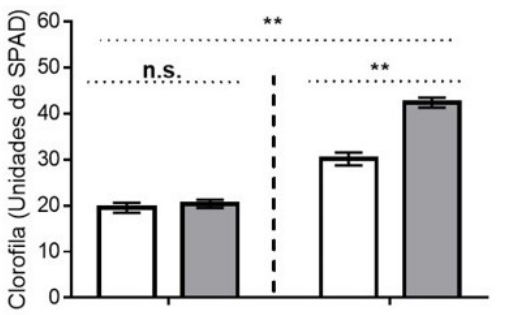

b)

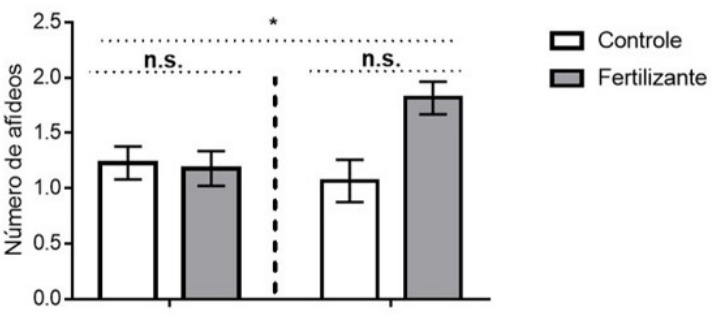

c)

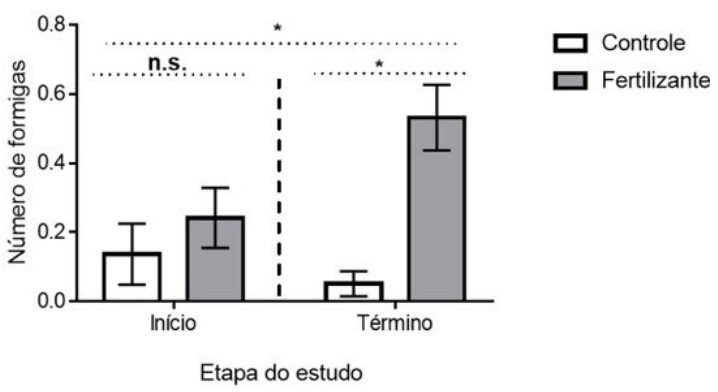

Figura 1. Efeitos (Média $\pm \mathrm{SE}$ ) de Tithonia diversifolia sem fertilização (Controle) e com fertilização (Fertilizante) ao início e término do estudo (12 dias) sobre (a) clorofila foliar (unidades de SPAD) da planta, (b) número de afídeos (Aphis gossypii) e (c) números de formigas (Solenopsis invicta e Pheidole megacephala) em um experimento em casa de vegetação. O número de afídeos e de formigas foram transformados em logaritmo. *, ** e n.s. indicam, respectivamente, $p$ $<0,001, p<0,05$ e $p>0,05$. 
Tabela 1. Resultado da ANOVA de medidas repetidas para a concentração de clorofila foliar (unidades de SPAD) de Tithonia diversifolia, número de formigas e número de Aphis gossypii em relação aos grupos de fertilização ao longo do tempo (12 dias) em experimento em casa de vegetação.

\begin{tabular}{lccccc}
\hline Variável & $\begin{array}{l}\text { Fonte de } \\
\text { variação }\end{array}$ & gl & QM & F & $\boldsymbol{p}$ \\
\hline Clorofila & Grupo & 1 & 643,73 & 24,27 & $\mathbf{0 , 0 0 1}$ \\
& Tempo & 1 & 3977,8 & 348,47 & $\mathbf{0 , 0 0 1}$ \\
& Grupo x Tempo & 1 & 490,03 & 42,93 & $\mathbf{0 , 0 0 1}$ \\
Número de afídeos & Erro & 28 & 319,62 & & \\
& Grupo & 1 & 0,84 & 2,74 & 0,097 \\
& Tempo & 1 & 1,83 & 3,81 & 0,061 \\
& Grupo x Tempo & 1 & 2,39 & 14,44 & $\mathbf{0 , 0 3 4}$ \\
Número de formigas & Erro & 28 & 0,48 & & \\
& Grupo & 1 & 1,28 & 19,02 & $\mathbf{0 , 0 0 2}$ \\
& Tempo & 1 & 0,16 & 1,55 & 0,224 \\
& Grupo x Tempo & 1 & 0,53 & 5,14 & $\mathbf{0 , 0 3 1}$ \\
& Erro & 28 & 0,1 & & \\
\hline
\end{tabular}

Resultados em negrito indicam $p<0,05$. Siglas: $\mathrm{gl}=$ grau de liberdade; $\mathrm{QM}=$ Quadrado Médio.

\section{Discussão}

A fertilização sintética de $T$. diversifolia aumenta a concentração de clorofila, porém esta condição não é suficiente para refletir no aumento número A. gossypii, apesar incrementar o número de formigas ( $S$. invicta e $P$. megacephala) que ordenham esses afídeos. A alta concentração de clorofila de $T$. diversifolia representa a grande capacidade de acumulação de nutrientes edáficos, uma característica comum em plantas daninhas, fato corroborado por Gioria \& Osborne (2014). A fertilização acelera o desenvolvimento de estruturas vegetativas do girassol-mexicano, além de aumentar a capacidade de invasão e tolerância à herbivoria (Venâncio, 2017).

O número de indivíduos de Aphis gossypii incrementa rapidamente em algumas plantas fertilizadas (Rostami et al., 2012). Nosso estudo não demostrou esta relação na interação do afídeos com $T$. diversifolia fertilizadas, porém o número de afídeos aumentaram em quase $100 \%$ para plântulas fertilizadas do início ao final do experimento, enquanto essas variáveis diminuíram na ausência de fertilizante. Esses resultados podem ser reflexo da curta duração do experimento (12 dias) e baixo número de réplicas de plântulas. Esta espécie de afídeo pode demorar até 10 dias para completar a fase de amadurecimento em algumas plantas hospedeiras (Ebert \& Cartwright, 1997; Saha, Singh \& Radhakrishore, 2009), o que pode ter ocorrido em $T$. diversifolia.

O maior número de formigas para as plantas fertilizadas indica que não houve uma relação densidade-dependente com A. gossypii, mas sim uma possível preferência às propriedades do honeydew liberado pelos afídeos (Cushman, 1991; Zhou et al., 2015). Afídeos que se alimentam de plantas ricas nutricionalmente liberam um honeydew mais energético e capaz de atrair quimicamente formigas mutualistas (Cushman, 1991; Zhou et al., 2015). Nossos resultados mostraram ainda que houve um aumento e decréscimo no número de formigas respectivamente nas plantas sem fertilização e fertilizadas, indicando a translocação de formigas de um grupo ao outro.

\section{Conclusão}

$\mathrm{O}$ estudo demonstrou que a fertilização pode desencadear um efeito bottom-up positivo na interação facultativa entre as espécies invasoras de plantações: girassol-mexicano, afídeo-do-algodão e formigas mutualistas (S. invicta e $P$. megacephala). Outros estudos devem replicar o uso de fertilizante em teias tróficas de invasoras para verificar como outras pragas agrícolas e de ambientes naturais se comportam sob o efeito desse insumo agrícola, além de contornar as limitações encontradas neste estudo.

\section{Agradecimentos}

Os autores agredecem ao Kassio Vinicio Moreira e à Maria Vitória Godoi pelo suporte logístico. Este estudo foi financiado pela CAPES, CNPq (Processos: 145872/2018-1 e 140158/2018-9) e DURATEX.

\section{Referências}

BACCARO, F. B.; FEITOSA, R. M.; FERNÁNDEZ, F.; FERNANDES, I. O.; IZZO, T. J.; SOUZA, J. L.; SOLAR, R. 2015. Guia para os gêneros de formigas do Brasil. Editora INPA, Manaus.

BUTLER, J.; GARRATT, M. P. D.; LEATHER, S. R. 2012. Fertilizers and insect herbivores: a metaanalysis. Ann. App. Biol., v. 161, n. 3, p. 223-233. 
CUSHMAN, J. H. 1991. Host-plant mediation of insect mutualisms: variable outcomes in herbivoreant interactions. Oikos, v. 61, n. 1, p. 138-144.

EBERT, T. A.; CARTWRIGHT, B. 1997. Biology and ecology of Aphis gossypii Glover (Homoptera: Aphididae). Southwest. Entomol., v. 22, n. 1, p. 116-153.

GIORIA, M.; OSBORNE, B. A. 2014. Resource competition in plant invasions: emerging patterns and research needs. Front. Plant Sci., v. 5, p. 1-21.

KAPLAN, I.; EUBANKS, M. D. 2002. Disruption of Cotton Aphid (Homoptera: Aphididae), Natural Enemy Dynamics by Red Imported Fire Ants (Hymenoptera: Formicidae). Environ. Entomol., v. 31, p. 1175-1183.

KOŁODZIEJEK, J. 2019. Growth and competitive interaction between seedlings of an invasive Rumex confertus and of co-occurring two native Rumex species in relation to nutrient availability. Sci. Rep., v. 9, n. 1, p. 1-12.

MORALES, M. A.; BEAL, A. L. H. 2006. Effects of host plant quality and ant tending for treehopper Publilia concava. Ann. Entomol. Soc. Am., v. 99, n. 3, p. 545-552.

NETTO, A. T.; CAMPOSTRINI, E.; OLIVEIRA, J. G.; BRESSAN-SMITH, R. E. 2005. Photosynthetic pigments, nitrogen, chlorophyll a fluorescence and SPAD-502 readings in coffee leaves. Sci. Hortic., v. 104, n. 2, p. 199-209.

ROSTAMI, M.; ZAMANI, A. A.; GOLDASTEH, S.; SHOUSHTARI, R. V.; KHERADMAND, K. K. K. 2012. Influence of Nitrogen Fertilization on Biology of Aphis gossypii (Hemiptera: Aphididae) Reared on Chrysanthemum lindicum (Asteraceae). J. Plant Prot. Res., v. 52, n. 1, p. 118-121.

SAHA, M. A. S.; SINGH, T. K.; RADHAKRISHORE, R. K. 2009. Comparative biology of cotton aphid, Aphis gossypii Glover
(Homoptera-Aphididae) on okra and brinjal. J. Exp. Zool., v. 12, n. 2, p. 373-375.

SINGH, V.; SOOD, A. K. 2017. Plant Nutrition: A tool for the management of hemipteran insectpests-A review. Agric. Rev., v. 38, n. 4, p. 260-270.

STANLEY, M. C.; H. W. NATHAN, L. K.; PHILLIPS, S. J.; KNIGHT, J. A.; GALBRAITH, C. J.; WINKS; WARD, D. F. 2013. Invasive interactions: Can Argentine ants indirectly increase the reproductive output of a weed? Arthropod Plant. Interact., v. 7, n. 1, p. 59-67.

TULLOSS, E. M.; CADENASSO, M. L. 2016. The Effect of Nitrogen Deposition on Plant Performance and Community Structure: Is It Life Stage Specific?. PloS one, v. 11, n. 6, e0156685.

VENÂNCIO, H.; SILVA, E. A.; SANTOS, J. C. 2016. Is a leaf life span enough to display changes on developmental instability and nitrogen after simulated herbivory? Flora, v. 222, p.121-127.

VENÂNCIO, H. 2017. Efeitos da herbivoria e condições ambientais sobre a instabilidade no desenvolvimento e resposta vegetativa em Tithonia diversifolia (Asteraceae). Uberlândia, Minas Gerais, Brasil. 102 p.

YAO, I. 2014. Costs and constraints in aphid-ant mutualism. Ecol. Res., v. 29, n. 3, p. 383-391.

ZHOU, A. M.; LU, Y. Y.; ZENG, L.; XU, Y. J.; LIANG, G. W. 2012. Does mutualism drive the invasion of two alien species? The case of Solenopsis invicta and Phenacoccus solenopsis. PloS One, v. 7, n. 7, e41856.

ZHOU, A. M.; WU, D.; LIANG, G. W.; Lu, Y. Y.; XU, Y. 2015. Effects of tending by Solenopsis invicta (Hymenoptera: Formicidae) on the sugar composition and concentration in the honeydew of an invasive mealybug, Phenacoccus solenopsis (Hemiptera: Pseudococcidae). Ethology, v. 121, n. 5, p. 492-500. 